\title{
SOLVING DUAL FUZZY NONLINEAR EQUATIONS VIA MODIFIED STIRLING'S METHOD
}

\author{
Umar.A. $\mathbf{O}^{1}$ \\ Faculty of Informatics and Computing, Universiti Sultan Zainal Abidin, (Unisza) Kuala Terengganu, \\ 21300, Terengganu, Malaysia. \\ (Email: umarabdul64@gmail.com) \\ Mamat. $\mathbf{M}^{2}$ \\ Faculty of Informatics and Computing, Universiti Sultan Zainal Abidin, (Unisza) Kuala Terengganu, \\ 21300, Terengganu, Malaysia. \\ (Email: must@unisza.edu.my) \\ Waziri. M.Y $\mathbf{Y}^{3}$ \\ Department of Mathematics, Bayero University, (Buk) Kano, Nigeria. \\ (Email: mywaziri@gmail.com)
}

Received date: $29-03-2019$

Revised date: 11-09-2019

Accepted date : 21-09-2019

Published date: 22-09-2019

To cite this document: Umar, A. O., Mamat, M., \& Waziri, M. Y. (2019). Solving Dual Fuzzy Nonlinear Equations Via Modified Striling's Method. Journal of Information System and Technology Management, 4(14), 84-91.

DOI: $10.35631 /$ jistm. 414008

Abstract: Stirling's method is a root finding method designed to approximate a locally unique fixed point and cannot be used to solve fuzzy nonlinear equations. In this paper we present a modified Stirling's method for solving dual fuzzy nonlinear equations. The fuzzy coefficient is presented in parametric form. Numerical results on some benchmark problems indicate that the proposed method is efficient.

Keywords: Fixed Point, Fuzzy Parametric Form, Dual Fuzzy Nonlinear Equation

\section{Introduction}

One of the most important problems in computational mathematics is solving nonlinear equations of the form

$$
F(x)=0 \text {. }
$$

The problem of solving these kinds of problems arises in diverse areas such as engineering, mathematics, computer science and social science. However, the parameters of these nonlinear equations are usually represented by fuzzy numbers rather than crisp numbers. Therefore, the solution depends on the parametric form of the fuzzy nonlinear equation. The concept of fuzzy numbers was first presented by (Zadeh, 1965). However, according to fuzzy arithmetic, dual fuzzy nonlinear system cannot be replaced by a fuzzy nonlinear system (Buckley \& Jowes, 2007). Also, analytic solutions are often not available for most type of fuzzy nonlinear 
equations and were such method exists, like (Buckley \& Qu,1990,1991) they are not suitable for solving systems such as

$$
\begin{aligned}
& \text { (1) } a x^{5}+b y^{4}+c x^{3}+d y^{3}+e x^{2} y^{2}+f=g \\
& \text { (2) } a x^{2}+b=d \\
& \text { (3) } x-\cos y=p
\end{aligned}
$$

where $a, b, c, d, e, f, g$ and $p$ are fuzzy numbers. Hence, numerical iterative methods are best suited for this kind of problems. However, most numerical methods considered require the computation and storage of either the Jacobian or approximate Jacobian at every iteration. For example, Abbasbandy and Asady (2004) consider newton's method for solving fuzzy nonlinear equation. Kajani, Asady and Vencheh (2005) apply newton's method for solving dual fuzzy nonlinear system. Newton's method is known to converge rapidly when the initial guess is chosen near the solution point (Wazir \& Moyi, 2016). However, the disadvantage of Newton's method is the need to compute and invert the Jacobian at every iteration. To overcome some of the shortcoming of Newton's method, various methods are employed,for example, Waziri and Moyi (2016) used chord method to solve dual fuzzy nonlinear equations. This method computes the Jacobian only once throughout the iteration process. Sulaiman mamat and Waziri (2018) used Shamanskii method for solving fuzzy nonlinear equations. This method starts by computing Newton's step in the first iteration, followed by several chord iterates. The reason is to reduce the computational cost of Jacobian evaluation at all iterations. Ramli, Abdullah and Mamat (2010) solved fuzzy nonlinear equations using Broyden's method. The method replaces the matrix Jacobian with a matrix $A_{k-1}$ whose inverse is directly determined at each iteration. However these methods are variants of Newton's method and share some of the drawback of Newton's method. Stirling's method (Rall1975, Argyros, 2008, and Parhi \& Gupta,2010) is designed to approximate a locally unique fixed point of the nonlinear equation.

The major drawback of this method is that it cannot be used to solve fuzzy nonlinear equations. In this paper a new approach via modified Stirling's method is proposed to solve dual fuzzy nonlinear equations. The proposed method extends the method to solving nonlinear equations. This paper is structured as follows. In section 2, we present some basic definition and fundamental results of fuzzy numbers. In section 3, we propose an iterative method for solving a dual fuzzy nonlinear equation. In section 4 , we present modified Stirling's method for solving dual fuzzy nonlinear equation. In section 5, we illustrate our method by some numerical examples. Conclusions are given in the last section.

\section{Preliminaries}

\section{Definition 1}

(Buckley \& $\mathrm{Qu}, 1991)$. A fuzzy number is a set like $u: R \rightarrow I=[0,1]$ which satisfy the following conditions,

(1) $u$ is upper semicontinuous,

(2) $u(x)=0$ outside some interval $[c, d]$,

(3) There are real numbers $a, b$ such that $c \leq a \leq b \leq d$ and

(3.1) $u(x)$ is monotonic increasing on $[c, a]$

(3.2) $u(x)$ is monotonic decreasing on $[b, d]$

(3.3) $u(x)=1, a \leq x \leq b$.

The set of all these fuzzy numbers is denoted by E. An equivalent parametric is also given in as follows (Buckley \& Qu, 1991). 


\section{Definition 2}

A fuzzy number $u$ in parametric form is a pair $(\underline{u}, \bar{u})$ of function $\underline{u}(r), \bar{u}(r), 0 \leq r \leq 1$, which satisfies the following requirements:

(1) $\underline{u}(r)$ is bounded monotonic increasing left continuous function,

(2) $\overline{\bar{u}}(r)$ is bounded monotonic decreasing left continuous function,

(3) $\underline{u}(r)<\bar{u}(r), 0 \leq r \leq 1$.

A popular fuzzy number is the trapezoidal fuzzy number $u=\left(x_{0}, y_{0}, \sigma, \beta\right)$ with interval defuzzifier $\left[x_{0}, y_{0}\right]$ and left fuzziness $\sigma$ and right fuzziness $\beta$ where the membership function is

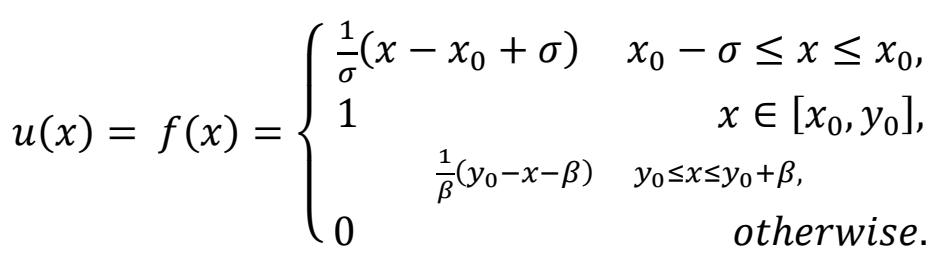

In parametric form we have $\underline{u}(r)=x_{0}-\sigma+\sigma r, \quad \bar{u}(r)=y_{0}+\beta-\beta r$.

Let $T F(R)$ be the set of all trapezoidal fuzzy numbers. The addition and scalar multiplication of fuzzy number are defined by the extension principle and can be equivalently represented as follows.

For arbitrary $u=(\underline{u}, \bar{u}), \quad v=(\underline{v}, \bar{v})$ and $k>0$ we define addition and multiplication by scalar $k$ as

$$
\begin{array}{ll}
(\underline{u+v})(r)=\underline{u}+\underline{v}, & (\overline{u+v)}(r)=\bar{u}+\bar{v}, \\
(\underline{k u})(r)=k \underline{u}(r), & (\overline{k u)}(r)=k \bar{u}(r),
\end{array}
$$

\section{Modified Stirling's Method (MSM)}

Stirling's method (Rall, 1975; Argyros, 2008) is designed to approximate a locally unique fixed point $x^{*}$ of a nonlinear equation

$$
F(x)=x
$$

We extend the method to solve dual fuzzy nonlinear equations of the form

$$
f(x)=0
$$

We propose a modification of Stirling's method for solving dual fuzzy nonlinear equation. The anticipation is to extend the linear root finding method to solve nonlinear equations. The proposed method is given as follows: The modified Stirling's method generates an iterative sequence $\left\{x_{n}\right\}$ via the following:

$$
x_{n+1}=x_{n}-\left[I-F^{1}\left(P\left(x_{n}\right)\right)\right]^{-1}\left(F\left(x_{n}\right)\right) \quad n=1,2 \ldots
$$

where $P\left(x_{n}\right)=x_{n}$. 


\section{Iterative Approach For Solving Dual Fuzzy Nonlinear Equations}

Computational literature shows that some basic operations from real numbers used to solve crisp equations do not hold for non-crisp (fuzzy) equations. In fact, for any non-crisp fuzzy number $x \in E$ (Waziri \& Moyi, 2016) it is true that

$$
x+(-x) \neq 0
$$

Hence, we consider the Dual Fuzzy nonlinear equation

$$
H(x)=R(x)+B
$$

where all the parameters are fuzzy numbers. Now our aim is to obtain a solution for the above dual fuzzy nonlinear equation; the parametric form is written as

$$
\begin{aligned}
& \underline{H}(\underline{x}, \bar{x}, r)=\underline{R}(\underline{x}, \bar{x}, r)+\underline{B}(r), \\
& \bar{H}(\bar{x}, \underline{x}, r)=\bar{R}(\bar{x}, \underline{x}, r)+\underline{B}(r) \quad \forall r \in[0,1]
\end{aligned}
$$

Now, let $x=(\underline{\alpha}, \bar{\alpha})$ be the solution to the above fuzzy nonlinear equation, then

$$
\begin{aligned}
& \underline{H}(\underline{\alpha}, \bar{\alpha}, r)=\underline{R}(\underline{\alpha}, \bar{\alpha}, r)+\underline{B}(r), \\
& \bar{H}(\bar{\alpha}, \underline{\alpha}, r)=\bar{R}(\bar{\alpha}, \underline{\alpha}, r)+\bar{B}(r) \quad \forall r \in[0,1]
\end{aligned}
$$

Rewriting (3) we obtain

$$
\begin{aligned}
& \underline{H}(\underline{\alpha}, \bar{\alpha}, r)-\underline{R}(\underline{\alpha}, \bar{\alpha}, r)=\underline{B}(r), \quad \\
& \bar{H}(\bar{\alpha}, \underline{\alpha}, r)-\bar{R}(\bar{\alpha}, \underline{\alpha}, r)=\bar{B}(r) \quad \forall r \in[0,1]
\end{aligned}
$$

Let $\underline{Q}(\underline{\alpha}, \bar{\alpha}, r)=\underline{B}(r)$ and $\bar{Q}(\bar{\alpha}, \underline{\alpha}, r)=\bar{B}(r)$ in (4), we get

$$
\begin{aligned}
& \underline{H}(\underline{\alpha}, \bar{\alpha}, r)-\underline{R}(\underline{\alpha}, \bar{\alpha}, r)=\underline{Q}(\underline{\alpha}, \bar{\alpha}, r), \\
& \bar{H}(\bar{\alpha}, \underline{\alpha}, r)-\bar{R}(\bar{\alpha}, \underline{\alpha}, r)=\bar{Q}(\bar{\alpha}, \underline{\alpha}, r) \quad \forall r \in[0,1]
\end{aligned}
$$

Hence, if $x_{k}=\left(\underline{x}_{k}, \bar{x}_{k}\right)$ is an approximate solution to the given system, then there exist $h(r)$ and $g(r)$ and $\forall r \in[0,1]$ such that

$$
\begin{aligned}
& \underline{\alpha}(r)=\underline{x}_{k}(r)+h(r) \\
& \bar{\alpha}(r)=\overline{\bar{x}}_{k}(r)+g(r), \quad k=0,1,2, \ldots
\end{aligned}
$$

Now if we use the Taylor series of $\underline{F}, \bar{F}$ about $\left(\underline{x}_{0}, \bar{x}_{0}\right)$, then for any $r \in[0,1]$,

$$
\begin{aligned}
& \underline{F}(\underline{\alpha}, \bar{\alpha}, r)=\underline{F}\left(\underline{x}_{0}, \bar{x}_{0}, r\right)+h \underline{F}_{\underline{x}}\left(\underline{x}_{0}, \bar{x}_{0}, r\right)+g \underline{F} \underline{x}_{\bar{x}}\left(\underline{x}_{0}, \bar{x}_{0}, r\right)+0\left(h^{2}+h k+h^{2}\right)=\underline{c} \\
& \bar{F}(\underline{\alpha}, \bar{\alpha}, r)=\bar{F}\left(\underline{x}_{0}, \bar{x}_{0}, r\right)+h \underline{F}_{\underline{x}}\left(\underline{x}_{0}, \bar{x}_{0}, r\right)+g \bar{F}_{\bar{x}}\left(\underline{x}_{0}, \bar{x}_{0}, r\right)+0\left(h^{2}+h k+h^{2}\right)=\bar{c}
\end{aligned}
$$

Eliminating the terms with highest order, and if $\underline{x}_{0}$ and $\bar{x}_{0}$ are near to $\underline{\alpha}$ and $\bar{\alpha}$, then $h(r)$ and $g(r)$ are small. We assume that all needed partial derivatives exist and are bounded. Therefore for enough small $h(r)$ and $g(r)$, then for each $r \in[0,1]$ 


$$
\begin{aligned}
& \underline{F}\left(\underline{x}_{0}, \bar{x}_{0}, r\right)+h \underline{F}_{\underline{x}}\left(\underline{x}_{0}, \bar{x}_{0}, r\right)+g \underline{F} \overline{\bar{x}}\left(\underline{x}_{0}, \bar{x}_{0}, r\right)=\underline{c}(r) \\
& \bar{F}\left(\underline{x}_{0}, \bar{x}_{0}, r\right)+h \bar{F}_{\underline{x}}\left(\underline{x}_{0}, \bar{x}_{0}, r\right)+g \bar{F} \bar{x}\left(\underline{x}_{0}, \bar{x}_{0}, r\right)=\bar{c}(r)
\end{aligned}
$$

And hence $h(r)$ and $g(r)$ can be obtained by solving the following equations.

In this paper $\forall r \in[0,1]$ we use modified Stirling's method to solve (10). The modified Stirling's method generates a sequence

$$
x_{n+1}=x_{n}-\left[I-F^{1}\left(P\left(x_{n}\right)\right)\right]^{-1}\left(F\left(x_{n}\right)\right) \quad n=1,2 \ldots
$$

Where $P\left(x_{n}\right)=x_{n}$ and $I$ an identity matrix and

$$
J\left(\underline{x}_{0}, \bar{x}_{0}, r\right)\left(\begin{array}{l}
h(r) \\
g(r)
\end{array}\right)=\left(\begin{array}{l}
\underline{c}(r)-\underline{F}\left(\underline{x}_{0}, \bar{x}_{0}, r\right) \\
\bar{c}(r)-\bar{F}\left(\underline{x}_{0}, \bar{x}_{0}, r\right)
\end{array}\right)
$$

Where

$$
J\left(\underline{x}_{0}, \bar{x}_{0}, r\right)=\left[\begin{array}{ll}
\underline{F}_{\underline{x}}\left(\underline{x}_{0}, \bar{x}_{0}, r\right) & \underline{F}_{\bar{x}}\left(\underline{x}_{0}, \bar{x}_{0}, r\right) \\
\bar{F}_{\underline{x}}\left(\underline{x}_{0}, \bar{x}_{0}, r\right) & \bar{F}_{\bar{x}}\left(\underline{x}_{0}, \bar{x}_{0}, r\right)
\end{array}\right]
$$

We now propose the algorithm for the proposed method

\section{Algorithm: Modified Stirling's Method (MSM)}

Step 1: Transform the dual fuzzy nonlinear equations into parametric form

Step 2: Determine the initial guess $x_{0}$ by solving the parametric equations for $r=0$ and $r=$ 1.

And for $n=0,1,2 \ldots$

Step 3: Compute $J_{n}\left(x_{n}\right)$ and $F\left(x_{n}\right)$. where $J_{n}\left(x_{n}\right)=F^{1}\left(P\left(x_{n}\right)\right)$

Step 4. Compute $\left[I-J_{0}\left(x_{n}\right)\right] s_{n}=-F\left(x_{n}\right)$. where I is an identity matrix

Step 5. Update $x_{n+1}=x_{n}+s_{n}$. where $s_{n}=-\left[I-J_{0}\left(x_{n}\right)\right]^{-1} F\left(x_{n}\right)$

Step 6: Repeat Step 3 to Step 5 and continue with the next $\mathrm{n}$ until $\left\|F\left(x_{n}\right)\right\| \leq 10^{-4}$ are satisfied.

\section{Numerical Applications}

Here we consider two examples to illustrate the performance of the iterative method for solving dual fuzzy nonlinear equation. The computation are done in MATLAB 2013a using double precision computer.

Example 1 (Buckley \& Qu, 1990) Consider a dual fuzzy nonlinear equation

$$
(3,4,5) x^{2}+(1,2,3) x-(1,2,3)=0
$$

Without loss of generality, let's assume $\mathrm{x}$ is positive. The parametric form of the above equation is as follows:

$$
\begin{aligned}
& (3+r) \underline{x}^{2}(r)+(1+r) \underline{x}(r)-(1+r)=0 \\
& (5-r) \bar{x}^{2}(r)+(3-r) \bar{x}(r)-(3-r)=0
\end{aligned}
$$


We solve the parametric form for $r=0$ and $r=1$ to obtain the initial guess.

i.e. for $r=1$ we have

$$
\begin{array}{r}
4 \underline{x}^{2}(1)+2 \underline{x}(1)=2 \\
4 \bar{x}^{2}(1)+2 \bar{x}(1)=2
\end{array}
$$

and for $r=0$, we have

$$
\begin{gathered}
3 x^{2}(0)+\underline{x}(0)=1 \\
5 \bar{x}^{2}(0)+3 \bar{x}(0)=3
\end{gathered}
$$

We have, $\underline{x}(0)=0.4343, \bar{x}(0)=0.5307$ and $\underline{x}(1)=\bar{x}(1)=0.5000$. Considering, initial guess as $x_{0}=(0.4,0.5)$, after three iterations, we obtain the solution with the maximum error less than $10^{-3}$. The performance profile is given in fig 1 .

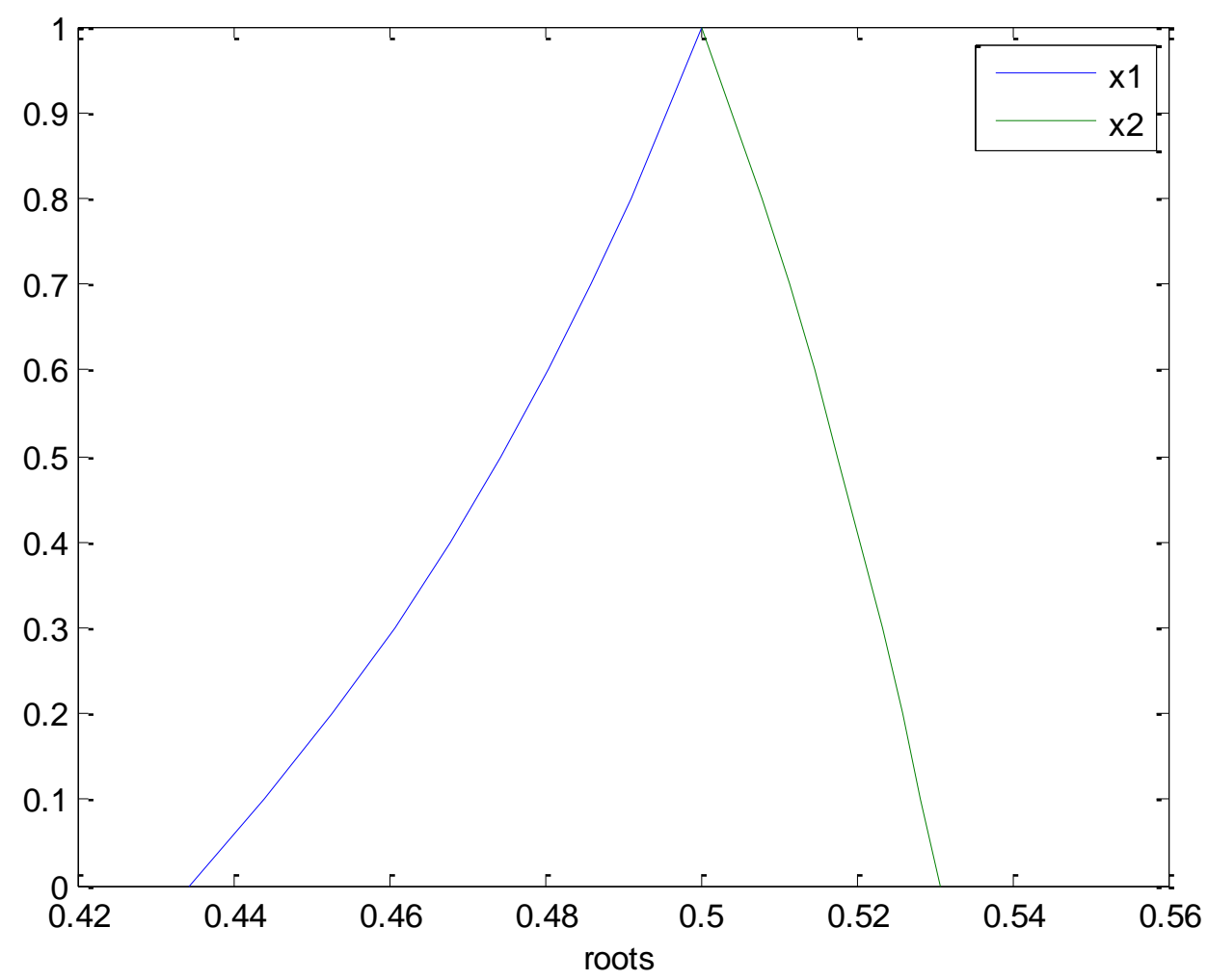

Figure 1: Solution of Stirling's Method For Example 1

Example 2 (Buckley \& Qu, 1990) Consider a dual fuzzy nonlinear equation

$$
(4,6,8) x^{2}+(2,3,4) x-(3,6,9)=0
$$

Without loss of generality, we assume that $\mathrm{x}$ is positive, and then the parametric form of the above equation is as follows:

$$
\begin{aligned}
& (4+2 r) \underline{x}^{2}(r)+(2+r) \underline{x}(r)-(3+3 r)=0 \\
& (8-2 r) \bar{x}^{2}(r)+(4-r) \bar{x}(r)-(9-3 r)=0
\end{aligned}
$$


We solve the parametric form for $r=0$ and $r=1$ to obtain the initial guess as follows: for $r=0$, we have

$$
\begin{aligned}
& (4) \underline{x}^{2}(0)+(2) \underline{x}(0)-3=0 \\
& (8) \bar{x}^{2}(0)+(4) \bar{x}(0)-9=0
\end{aligned}
$$

and for $r=1$, we have

$$
\begin{aligned}
& (6) \underline{x}^{2}(1)+(3) \underline{x}(1)-6=0 \\
& (6) \bar{x}^{2}(1)+(3) \bar{x}(1)-6=0
\end{aligned}
$$

which implies $\underline{x}(0)=0.6514, \bar{x}(0)=0.8397$ and $\underline{x}(1)=\bar{x}(1)=0.7808$ and that the initial guess $x_{0}=(0.6514,0.7808,0.8397)$. The solution of the above system was obtained after four iterations with maximum error less than $10^{-3}$. Refer to fig 2 for the performance profile.

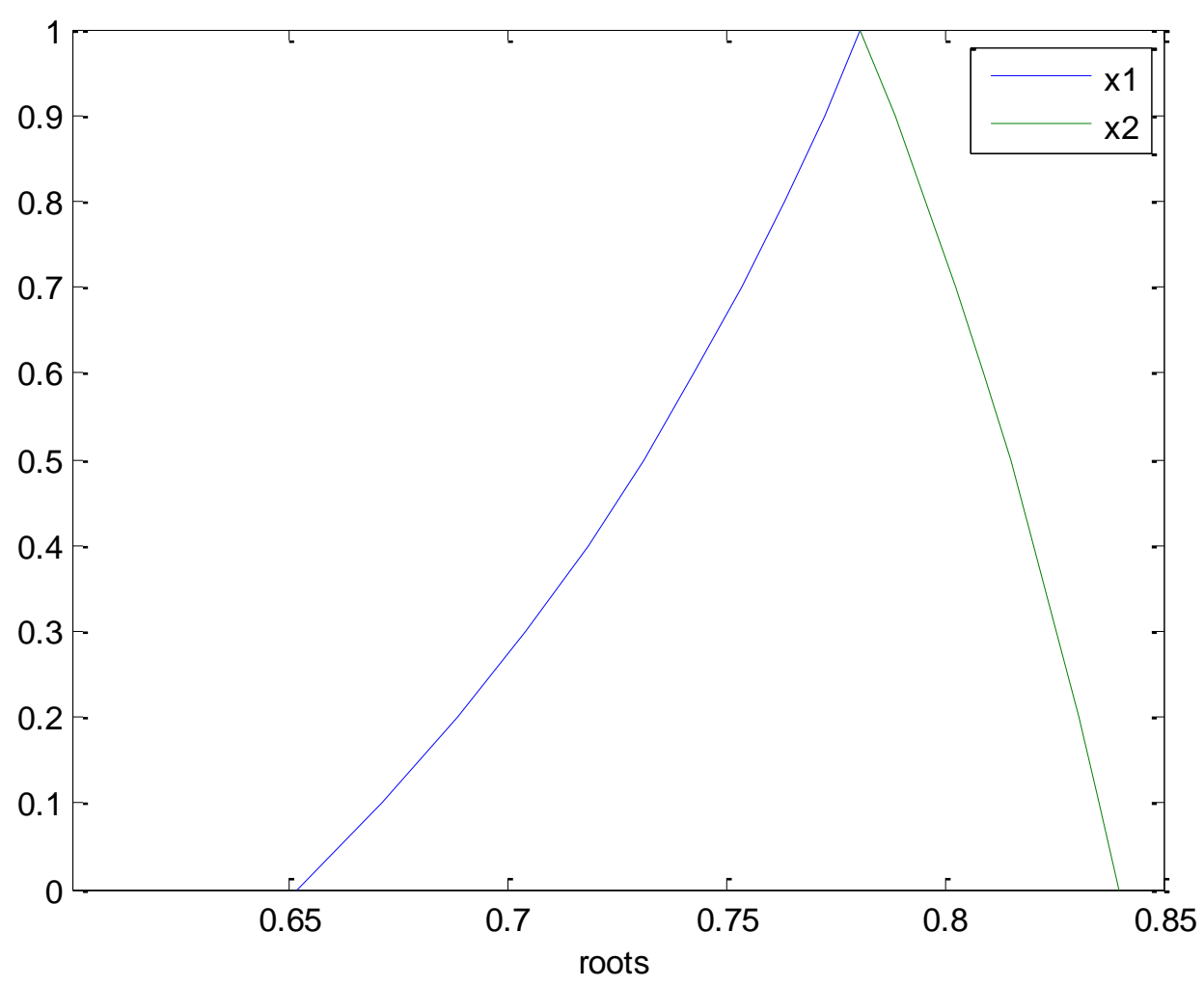

Figure 2: Solution of Stirling's Method for Example 2

\section{Conclusion}

In this paper, we proposed a modified Stirling's method for solving dual fuzzy nonlinear equations. The method is an extension of Stirling's method for root finding problems. The fuzzy nonlinear equations are written in parametric form and then solved via modified Stirling's method. Numerical examples for fuzzy nonlinear equations are provided to illustrate the efficiency of the proposed method, which shows that our proposed method is promising.

\section{References}

Abbasbandy, S., \& Asady, B. (2004). Newton's method for solving fuzzy nonlinear equations. Applied Mathematics and Computation, 159, 349-356. 
Argyros, I. K. (2008). Convergence and applications of Newton-type iterations. Springer Science \& Business Media.

Brent, R. P. (1973). Some efficient algorithms for solving systems of nonlinear equations. SIAM Journal on Numerical Analysis, 10, 327-344.

Buckley, J. J., \& Jowers, L. J. (2007). Solving fuzzy equations. In Monte Carlo Methods in Fuzzy Optimization (pp. 89-115). Springer, Berlin, Heidelberg.

Buckley, J. J., \& Qu, Y. (1990). Solving linear and quadratic fuzzy equations. Fuzzy sets and systems, 38, 43-59.

Buckley, J. J., \& Qu, Y. (1991). Solving systems of linear fuzzy equations. Fuzzy sets and systems, 43, 33-43.

Kajani, M. T., Asady, B., \& Vencheh, A. H. (2005). An iterative method for solving dual fuzzy nonlinear equations. Applied Mathematics and Computation, 167, 316-323.

Parhi, S. K., \& Gupta, D. K. (2010). Semilocal convergence of a Stirling-like method in Banach spaces. International Journal of Computational Methods, 7, 215-228.

Rall, L. B. (1975). Convergence of Stirling's method in Banach spaces. aequationes mathematicae, 12, 12-20.

Ramli, A., Abdullah, M. L., \& Mamat, M. (2010). Broyden's method for solving fuzzy nonlinear equations. Advances in Fuzzy Systems, 2010.

Shamanskii, V. E. (1967). A modification of Newton's method, Ukrain. Mat. Zh., 19.

Sulaiman, I.M.,Mamat,M. and Waziri,M.Y. (2018) Shamanskii method for Solving Fuzzy Nonlinear Equation. Proc. of the 2nd IEEE Intl Conf on Intl Syst Engr (ICISE) 20-21

Waziri, M. Y., \& Moyi, A. U. (2016). An alternative approach for solving dual fuzzy nonlinear equations. International Journal of Fuzzy Systems, 18, 103-107.

Yang, W. Y., Cao, W., Chung, T. S., \& Morris, J. (2005). Applied numerical methods using $M A T L A B$. John Wiley \& Sons.

Zadeh, L. A. (1965). Fuzzy sets. Information and control, 8, 338-353. 